\title{
Research Article \\ On Pointlike Interaction between Three Particles: Two Fermions and Another Particle
}

\author{
Robert Adol'fovich Minlos \\ Institute for Information Transmission Problems, Russian Academy of Sciences, \\ Bolshoy Karetnyi 19, Moscow, Russia \\ Correspondence should be addressed to Robert Adol'fovich Minlos, minl@iitp.ru \\ Received 3 April 2012; Accepted 29 April 2012 \\ Academic Editors: J. Banasiak and M. Martins
}

Copyright (C) 2012 Robert Adol'fovich Minlos. This is an open access article distributed under the Creative Commons Attribution License, which permits unrestricted use, distribution, and reproduction in any medium, provided the original work is properly cited.

The problem of construction of self-adjoint Hamiltonian for quantum system consisting of three pointlike interacting particles (two fermions with mass 1 plus a particle of another nature with mass $m>0$ ) was studied in many works. In most of these works, a family of one-parametric symmetrical operators $\left\{H_{\varepsilon}, \varepsilon \in \mathbb{R}^{1}\right\}$ is considered as such Hamiltonians. In addition, the question about the self-adjointness of $H_{\varepsilon}$ is equivalent to the one concerning the self-adjointness of some auxiliary operators $\left\{\tau_{l}, l=0,1, \ldots\right\}$ acting in the space $L_{2}\left(\mathbb{R}_{+}^{1}, r^{2} d r\right)$. In this work, we establish a simple general criterion of self-adjointness for operators $\tau_{l}$ and apply it to the cases $l=0$ and $l=1$. It turns out that the operator $\tau_{l=0}$ is self-adjoint for any $m$, while the operator $\tau_{l=1}$ is self-adjoint for $m>m_{0}$, where the value of $m_{0}$ is given explicitly in the paper.

\section{Introduction and Statement of the Problem}

This paper is continuation of works [1-4] studying the problem of construction of Hamiltonian for a quantum system which consists of two fermions with mass 1 interacting pointwise with a particle of another nature having mass $m$.

Originally, the construction of such Hamiltonian begins with introduction of the symmetric operator:

$$
H_{0}=-\frac{1}{2}\left(\frac{1}{m} \Delta_{y}+\Delta_{x_{1}}+\Delta_{x_{2}}\right)
$$

acting in a Hilbert space $\mathscr{H}=L_{2}\left(\mathbb{R}^{3}\right) \otimes L_{2}^{\text {asym }}\left(\mathbb{R}^{3} \times \mathbb{R}^{3}\right)$. Here, $x_{1}, x_{2} \in \mathbb{R}^{3}$ are the positions of fermions, $y$ is the position of a separate particle, and $\Delta_{y}, \Delta_{x_{1}}$, and $\Delta_{x_{2}}$ are Laplacians with respect to $y, x_{1}$, and $x_{2}$, respectively. The domain of definition of $H_{0}, D\left(H_{0}\right) \subset \mathscr{d}$ consists 
of smooth rapidly decreasing functions $\psi\left(y, x_{1}, x_{2}\right) \in \mathscr{H}$ on infinity, antisymmetrical with respect to $x_{1}, x_{2}$ and satisfying the following conditions:

$$
\left.\psi\left(y, x_{1}, x_{2}\right)\right|_{x_{i}=y}=0, \quad i=1,2 .
$$

Usually, some family $\left\{H_{\varepsilon}, \varepsilon \in \mathbb{R}^{1}\right\}$ of symmetric extensions of the operator $H_{0}$ is proposed as a possible "true" Hamiltonian of the system (the so-called Ter-Martirosian-Skornyakov extensions, see [5]). These extensions were constructed in [1-4]. For some values of mass $m$, the extensions of Ter-Martirosian-Skornyakov are self-adjoint (for all values of the parameter $\varepsilon)$; however, for the other values of $m$ they are only symmetric with nonzero deficiency indexes (equal for all $\varepsilon$ ). It turns out (see [3]) that the self-adjointness of all operators $\left\{H_{\varepsilon}\right\}$ is equivalent to the one for some auxiliary symmetric operator $\tau$ acting in the space $L_{2}\left(\mathbb{R}^{3}\right)$ (see below). This operator commutes with the operators $\left\{U_{g}, g \in O_{3}\right\}$ of the representation of the rotation group $O_{3}$ that acts in $L_{2}\left(\mathbb{R}^{3}\right)$ by the usual formula:

$$
\left(U_{g} f\right)(k)=f\left(g^{-1} k\right), \quad g \in O_{3}, f \in L_{2}\left(\mathbb{R}^{3}\right) .
$$

Let us denote by $\mathscr{\ell}_{l} \subset L_{2}\left(\mathbb{R}^{3}\right)$ the maximal subspace, where the representation (1.3) is multiplied by the irreducible representation of $O_{3}$ with weight $l, l=0,1,2, \ldots$ (see [6]). Evidently, the space $\mathscr{H}_{l}$ is invariant with respect to the operator $\tau$, and the restriction $\tau_{l}=\left.\tau\right|_{\mathscr{l}_{l}}$ of this operator to the space $\mathscr{d}_{l}$ is symmetric operator. The operator $\tau$ is selfadjoint if all the operators $\left\{\tau_{l}, l=0,1, \ldots\right\}$ are self-adjoint. In this paper, we find general simple conditions of self-adjointness of $\tau_{l}$ and the form of the defect subspaces (with small exclusions) when these conditions are broken. Then, we apply these conditions to the cases $l=0$ and $l=1$ and get that the operator $\tau_{l=0}$ is self-adjoint for all values of $m>0$, while the operator $\tau_{l=1}$ is self-adjoint for $m>m_{0}$ and has nonzero deficiency indexes for $m \leq m_{0}$, the constant $m_{0}>0$ is indicated below (see (5.4)).

By the way, we note that the value of $m_{0}$ obtained in this paper differs from that one given by mistake in [2].

\section{A Short Explanation of the Constructions from Papers [1-3]}

(1) After Fourier transformation:

$$
\begin{aligned}
\psi\left(y, x_{1}, x_{2}\right) & \longrightarrow \widetilde{\psi}\left(q, k_{1}, k_{2}\right) \\
& =\frac{1}{2 \pi^{9 / 2}} \int_{\left(\mathbb{R}^{3}\right)^{3}} \psi\left(y, x_{1} x_{2}\right) \exp \left\{-i(q, y)-i\left(k_{1}, x_{1}\right)-i\left(k_{2}, x_{2}\right)\right\} d y d x_{1} d x_{2} \\
& \equiv(\mp \psi)\left(q, k_{1}, k_{2}\right),
\end{aligned}
$$

and change of variables:

$$
P=q+k_{1}+k_{2}, \quad p_{j}=\frac{P}{m+2}-k_{j}, \quad j=1,2,
$$


the operator

$$
\widetilde{H}_{0}=\mp H_{0} \Psi^{-1},
$$

can be represented as a tensor sum:

$$
\widetilde{H}_{0}=\widetilde{H}_{0}^{(1)}+\frac{m}{m+1} \widetilde{H}_{0}^{(2)}
$$

where $H_{0}^{(1)}$ is a self-adjoint operator in $L_{2}\left(\mathbb{R}^{3}\right)$ :

$$
\left(\widetilde{H}_{0}^{(1)} f\right)(P)=\frac{P^{2}}{m+2} f(P), \quad P \in \mathbb{R}^{3}, f \in L_{2}\left(\mathbb{R}^{3}\right),
$$

and $\widetilde{H}_{0}^{(2)}$ acts in $L_{2}^{\text {asym }}\left(\mathbb{R}^{3} \times \mathbb{R}^{3}\right)$ by formula

$$
\left(\widetilde{H}_{0}^{(2)} g\right)\left(p_{1}, p_{2}\right)=G\left(p_{1}, p_{2}\right) g\left(p_{1}, p_{2}\right), \quad g \in L_{2}^{\text {asym }}\left(\mathbb{R}^{3} \times \mathbb{R}^{3}\right),
$$

with

$$
G\left(p_{1}, p_{2}\right)=p_{1}^{2}+p_{2}^{2}+\frac{2}{m+1}\left(p_{1}, p_{2}\right)>0
$$

The operator $\widetilde{H}_{0}^{(2)}$ is symmetric, and its domain is

$$
D\left(\widetilde{H}_{0}^{(2)}\right)=\left\{g \in L_{2}^{\text {asym }}\left(\mathbb{R}^{3} \times \mathbb{R}^{3}\right): \int_{\mathbb{R}^{3}} g\left(p_{1}, p_{2}\right) d p_{j}=0, j=1,2\right\}
$$

(2) the deficiency subspace $\mathcal{R}_{-1} \subset L_{2}^{\text {asym }}\left(\mathbb{R}^{3} \times \mathbb{R}^{3}\right)$ of the operator $\widetilde{H}_{0}^{(2)}$ consists of the functions of the form:

$$
U_{\mathscr{\delta}}\left(p_{1}, p_{2}\right)=\frac{\wp\left(p_{1}\right)-\wp\left(p_{2}\right)}{G\left(p_{1}, p_{2}\right)+1}
$$

where the function $\wp(p)$ belongs to Hilbert space

$$
\mathcal{L}=\left\{\wp: \int_{\mathbb{R}^{3}} \frac{|\wp(p)|^{2}}{\sqrt{p^{2}+1}} d p<\infty\right\},
$$

with inner product

$$
\left\langle\wp_{1}, \wp_{2}\right\rangle=\left(U_{\wp_{1}}, U_{\wp_{2}}\right)_{L_{2}\left(\mathbb{R}^{3} \times \mathbb{R}^{3}\right)} \equiv\left(W \wp_{1}, \wp_{2}\right)_{L_{2}\left(\mathbb{R}^{3}\right)}
$$


Here $W$ is some positive operator acting in $L_{2}\left(\mathbb{R}^{3}\right)$ (see [3]). The domain of the operator $\left(\widetilde{H}_{0}^{(2)}\right)^{*}$, that is, a conjugate to $\widetilde{H}_{0}^{(2)}$, is

$$
D\left(\left(\widetilde{H}_{0}^{(2)}\right)^{*}\right)=\left\{g \in L_{2}^{\text {asym }}\left(\mathbb{R}^{3} \times \mathbb{R}^{3}\right): g\left(p_{1}, p_{2}\right)=f\left(p_{1}, p_{2}\right)+U_{\mathscr{\ell}}\left(p_{1}, p_{2}\right)+\frac{U_{\psi}\left(p_{1}, p_{2}\right)}{G\left(p_{1}, p_{2}\right)+1}\right\}
$$

where $f \in D\left(\widetilde{H}_{0}^{(2)}\right), \wp, \psi \in \mathcal{L}$. In addition, the operator $\left(\widetilde{H}_{0}^{(2)}\right)^{*}$ acts by the formula:

$$
\left(\left(\widetilde{H}_{0}^{(2)}\right)^{*} g\right)\left(p_{1}, p_{2}\right)=G\left(p_{1}, p_{2}\right) g\left(p_{1}, p_{2}\right)-\left(\wp\left(p_{1}\right)-\wp\left(p_{2}\right)\right)
$$

where $\wp$ is defined by $(2.12)$.

The following asymptotics holds for vectors $g \in D\left(\left(\widetilde{H}_{0}^{(2)}\right)^{*}\right) N \rightarrow \infty$ :

$$
\int_{\left|p_{1}\right|<N} g\left(p_{1}, p_{2}\right) d p_{1}=4 \pi N_{\wp}\left(p_{2}\right)+b\left(p_{2}\right)+o(1)
$$

Here

$$
b(p)=-(T \wp)(p)+(W \psi)(p)
$$

where the operator $W$ is defined in $(2.11)$, and $(T \&)(p)$ is given by the following expression $(\mu=2 /(m+1))$

$$
(T \wp)(p)=2 \pi^{2} \sqrt{\left(1-\frac{\mu^{2}}{4}\right) p^{2}+1} \wp(p)+\int_{\mathbb{R}^{3}} \frac{\wp(t)}{G(t, p)+1} d t
$$

defined on the set:

$$
D(T)=\left\{\wp \in L_{2}\left(\mathbb{R}^{3}\right):|p| \varnothing(p) \in L_{2}\left(\mathbb{R}^{3}\right)\right\}
$$

The above-mentioned Ter-Martirosian-Skornyakov's extension $\widetilde{H}_{\varepsilon}^{(2)}$ of the operator $\widetilde{H}_{0}^{(2)}$ is obtained by requiring

$$
b(p)=\varepsilon \mathscr{\&}(p)
$$

where $\varepsilon \in \mathbb{R}^{1}$ is an arbitrary parameter.

Lemma 2.1. The operator $T$ defined in the space $L_{2}\left(\mathbb{R}^{3}\right)$ by $(2.16)$ is symmetric, and the selfadjointness of the operators $H_{\varepsilon}$ (for all $\varepsilon$ ) is equivalent to the self-adjointness of the operator $T$ (see $[2,3,5])$. 
The operator $T$ can be represented as a sum of two operators:

$$
T=\tau+T^{\prime}
$$

where the symmetric operator $\tau$ (with the domain $D(\tau)=D(T)$ ) acts as follows:

$$
(\tau \wp)(p)=2 \pi^{2} \sqrt{1-\frac{\mu^{2}}{4}}|p| \wp(p)+\int_{\mathbb{R}^{3}} \frac{\wp(t) d t}{G(t, p)}
$$

and $T^{\prime}$ is a bounded self-adjoint operator. Since the deficiency indexes of $T$ coincide with the ones of $\tau$ (see [7]), we shall study the conditions of self-adjointness for the operator $\tau$;

(3) as we said, the space $\mathscr{L}_{l} \subset L_{2}\left(\mathbb{R}^{3}\right)$ is invariant with respect to $\tau$; it has the form:

$$
\mathscr{\ell}_{l}=L_{2}\left(\mathbb{R}_{+}^{1}, r^{2} d r\right) \otimes L_{2}^{l}(S),
$$

where $L_{2}^{l}(S) \subset L_{2}(S)$ is the space of spherical functions of weight $l$ (see [6]) on the unit sphere $S \subset \mathbb{R}^{3}$. In addition, the operator $\tau_{l}=\left.\tau\right|_{\mathscr{\ell}_{l}}$ has the form

$$
\tau_{l}=M_{l} \otimes E_{l}
$$

where $E_{l}$ is the unit operator in $L_{2}^{l}(s)$, and $M_{l}$ acts in $L_{2}\left(\mathbb{R}_{+}^{1}, r^{2} d r\right)$ by the formula:

$$
\left(M_{l} f\right)(r)=2 \pi^{2} \sqrt{1-\frac{\mu^{2}}{4}} r f(r)+2 \pi \int_{-1}^{1} d x P_{l}(x) \int_{0}^{\infty} \frac{\left(r^{\prime}\right)^{2} f\left(r^{\prime}\right) d r^{\prime}}{r^{2}+\left(r^{\prime}\right)^{2}+\mu r r^{\prime} x}
$$

on the domain

$$
D\left(M_{l}\right) \equiv V=\left\{u \in L_{2}\left(\mathbb{R}_{+}^{1}, r^{2} d r\right): r u(r) \in L_{2}\left(\mathbb{R}_{+}^{1}, r^{2} d r\right)\right\}
$$

Here $P_{l}(x), l=0,1,2, \ldots, x \in[-1,1]$, are orthogonal polynomials (Legendre polynomials) satisfying $P_{l}(1)=1$ :

$$
P_{l}(x)=\frac{1}{2^{l} l !} \frac{d^{l}}{d x^{l}}\left(x^{2}-1\right)^{l}, \quad x \in(-1,1)
$$

The operators $\left\{M_{l}, l=0,1, \ldots\right\}$ are symmetric in $L_{2}\left(\mathbb{R}_{+}^{1}, r^{2} d r\right)$, and the self-adjointness of $M_{l}$ is equivalent to the self-adjointness of $\tau_{l}$. Later on, we shall study the operators $M_{l}$ and derive a condition of self-adjointness. 


\section{Preparatory Constructions}

For every function $u \in V \subset L_{2}\left(\mathbb{R}_{+}^{1}, r^{2} d r\right)$, we consider the family of functions

$$
\mathfrak{h}(u)=\left\{u_{\alpha}=r^{\alpha} u, \alpha \in[0,1], u_{0}=u\right\}
$$

which we call a chain (with initial element $u=u_{0}$ and the final one $u_{1}$ ). All functions $u_{\alpha} \in \mathfrak{h}(u)$ belong to $L_{2}\left(\mathbb{R}_{+}^{1}, r^{2} d r\right)$ and have a uniformly bounded norm:

$$
\left\|u_{\alpha}\right\|^{2} \leq\left\|u_{0}\right\|^{2}+\left\|u_{1}\right\|^{2}, \quad \alpha \in[0,1]
$$

Consider the unitary map (Mellin's transformation [8]):

$$
\omega: L_{2}\left(\mathbb{R}_{+}^{1}, r^{2} d r\right) \longrightarrow L_{2}\left(\mathbb{R}^{1}, d s\right): f(r) \longrightarrow \tilde{f}(s)=\frac{1}{\sqrt{2 \pi}} \int_{0}^{\infty} r^{-i s+1 / 2} f(r) d r, \quad s \in \mathbb{R}^{1}
$$

and its inverse:

$$
\left(\omega^{-1} \tilde{f}\right)(r)=\frac{1}{\sqrt{2 \pi}} \int_{-\infty}^{\infty} r^{i s-3 / 2} \tilde{f}(s) d s
$$

For every set of functions $B \subset L_{2}\left(\mathbb{R}_{+}^{1}, r^{2} d r\right)$, we denote by $\widetilde{B} \subset L_{2}\left(\mathbb{R}^{1}, d s\right)$ the set of their Mellin's transformations:

$$
\widetilde{B}=\omega B
$$

For every chain $\mathfrak{h}(u)$, we denote by $\Gamma_{u}$ the family of functions:

$$
\Gamma_{u}=\widetilde{\mathfrak{h}(u)}=\left\{\gamma_{\alpha}(s), \alpha \in[0,1]\right\}
$$

where $\gamma_{\alpha}(s)=\left(\omega u_{\alpha}\right)(s), u_{\alpha} \in \mathfrak{h}(u)$. The family $\Gamma_{u}$ can be represented as a function $\Gamma_{u}(z)$ of a complex variable $z=s+i \alpha$ in the strip:

$$
\begin{gathered}
I=\left\{z \in \mathbb{C}^{1}: \Im z \in[0,1]\right\}, \\
\Gamma_{u}(z)=\frac{1}{\sqrt{2 \pi}} \int_{0}^{\infty} r^{-i s-1 / 2+\alpha} u(r) d r=\frac{1}{\sqrt{2 \pi}} \int_{0}^{\infty} r^{-i z} u(r) d r .
\end{gathered}
$$

The function $\Gamma_{u}$ is said to be associated with the chain $\mathfrak{h}(u)$, and its values $\left\{\gamma_{\alpha}(s)\right\}$ on the lines $\xi_{\alpha}=\left\{z=s+i \alpha, s \in \mathbb{R}^{1}, 0 \leq \alpha \leq 1\right\} \subset I$ are called the sections of $\Gamma_{u}$. 
Proposition 3.1. For every chain $\mathfrak{h}(u), u \in V$, the associated function $\left\{\Gamma_{u}(z), z \in I\right\}$ is continuous in a closed strip $I$ and analytic inside this strip. Moreover, its sections $\left\{\gamma_{\alpha}\right\}$ satisfy the following inequality:

$$
\sup _{0 \leq \alpha \leq 1}\left\|\gamma_{\alpha}(\cdot)\right\|_{L_{2}\left(\mathbb{R}^{1}\right)}<\infty
$$

Inversely, any function $\{\Gamma(z), z \in I\}$ which possesses these properties is associated with some (unique) chain $\mathfrak{h}(v): \Gamma=\Gamma_{v}, v \in V$. Let call this chain generated by $\Gamma$. In addition, the functions $\left\{v_{\alpha}, \alpha \in\right.$ $[0,1]\}$ of the chain $\mathfrak{h}(v)$ are obtained by the inverse Mellin's transformation from the sections of $\Gamma=\left\{\gamma_{\alpha}\right\}$ :

$$
v_{\alpha}=\omega^{-1} \gamma_{\alpha}
$$

The proof of this proposition can be obtained by using the arguments given in the book by Paley and Wiener (see [9], Chapter I), which are related to the Fourier transformation of functions analytical in a strip in a complex plane. It is not difficult to reformulate these arguments in terms of Mellin's transformation.

Note that the estimate (3.8) for $\left\{\gamma_{\alpha}\right\}$ follows from the estimate (3.2) and the unitary Mellin's transformation. Denote by $\mathcal{G}$ a linear space of functions $\Gamma$ satisfying conditions of Proposition 3.1. Let us introduce two maps:

$$
\Omega: \mathfrak{h}(u) \longrightarrow \Gamma_{u} \in \mathcal{G}, \quad \Omega^{-1}: \Gamma_{u} \longrightarrow \mathfrak{h}(u)
$$

Let $N(z), z \in I$, be a bounded, continuous function in the strip $I$, which is analytic inside $I$. This function generates the family $\tilde{\kappa}_{\alpha}^{N}, \alpha \in[0,1]$ of bounded operators in $L_{2}\left(\mathbb{R}^{1}\right)$ which act as multiplication on the functions $n_{\alpha}^{N}(s)=\left.N(z)\right|_{z=s+i \alpha}, s \in \mathbb{R}^{1}, 0 \leq \alpha \leq 1$ :

$$
\left(\tilde{\kappa}_{\alpha}^{N} \psi\right)(s)=n_{\alpha}^{N}(s) \psi(s), \quad \psi \in L_{2}\left(\mathbb{R}^{1}\right) .
$$

Evidently, for any $\Gamma \in \mathcal{G}$, the function $N(z) \Gamma(z)$ belongs to $\mathcal{G}$. If the chain $\mathfrak{h}(u)$ is generated by $\Gamma=\Gamma_{u}$ and the chain $\mathfrak{h}(v)$ is generated by $N(z) \Gamma(z)=\Gamma_{v}(z)$, then

$$
v_{\alpha}=\kappa_{\alpha}^{N} u_{\alpha}, \quad \alpha \in[0,1], \quad u_{\alpha} \in \mathfrak{h}(u),
$$

where

$$
\kappa_{\alpha}^{N}=\omega^{-1} \widetilde{\kappa}_{\alpha}^{N} \omega
$$

Denote by $\Pi$ the following self-adjoint operator in $L_{2}\left(\mathbb{R}_{+}^{1}, r^{2} d r\right)$ :

$$
(\Pi f)(r)=r f(r)
$$

with the domain $D(\Pi)=V$. 
It is clear that for any $u \in V$, the power $\Pi^{\beta}, 0 \leq \beta \leq 1$ of the operator $\Pi$ is applicable to an element $u_{\alpha} \in \mathfrak{h}(u)$ if $\beta+\alpha \leq 1$ and

$$
\Pi^{\beta} u_{\alpha}=u_{\alpha+\beta}
$$

For the function $\Gamma_{u}$ that is associated with $\mathfrak{h}(u)$, the action of the operator $\tilde{\Pi}^{\beta}=\omega \Pi^{\beta} \omega^{-1}$ on the sections $\left\{\gamma_{\alpha}\right\}$ of $\Gamma_{u}$ has the form:

$$
\tilde{\Pi}^{\beta} \gamma_{\alpha}=\gamma_{\alpha+\beta}
$$

(again if $\alpha+\beta \leq 1$ ).

\section{The Operator $M_{l}$}

The operator $M_{l}$ (see (2.23)) can be represented as

$$
M_{l}=\Pi^{1 / 2} \kappa^{l} \Pi^{1 / 2},
$$

where $\kappa^{l}=\kappa_{1 / 2}^{l}$ is an operator in $L_{2}\left(\mathbb{R}_{+}^{1}, r^{2} d r\right)$ acting by the formula:

$$
\left(\kappa_{1 / 2}^{l} f\right)(r)=2 \pi^{2} \sqrt{1-\frac{\mu^{2}}{4}} f(r)+2 \pi \int_{-1}^{1} d x P_{l}^{0}(x) \int_{0}^{\infty} \frac{\left(r^{\prime}\right)^{2} f\left(r^{\prime}\right) d r^{\prime}}{\left(r r^{\prime}\right)^{1 / 2}\left(r^{2}+\left(r^{\prime}\right)^{2}+\mu x r r^{\prime}\right)} .
$$

Lemma 4.1. Operator $\kappa_{1 / 2}^{l}$ is bounded and self-adjoint in $L_{2}\left(\mathbb{R}_{+}^{1}, r^{2} d r\right)$.

Proof. Pass to the operator:

$$
\tilde{\kappa}_{1 / 2}^{l}=\omega \kappa_{1 / 2}^{l} \omega^{-1}
$$

acting in $L_{2}\left(\mathbb{R}^{1}\right)$. It follows from calculations in $[2,3]$ that $\tilde{\kappa}_{1 / 2}^{l}$ is the operator of multiplication on the function:

$$
n_{1 / 2}^{l}(s)=2 \pi^{2}\left(\sqrt{1-\frac{\mu^{2}}{4}}+\lambda_{1 / 2}^{l}(s)\right)
$$

where

$$
\lambda_{1 / 2}^{l}(s)= \begin{cases}\int_{0}^{1} P_{l}(x) \frac{\operatorname{ch}(s v(x)) d x}{\operatorname{ch}(s \pi / 2) \cos (v(x))} & \text { for even } l, \\ -\int_{0}^{1} P_{l}(x) \frac{\operatorname{sh}(s v(x)) d x}{\operatorname{sh}(s \pi / 2) \cos (v(x))} & \text { for odd } l\end{cases}
$$


and $v(x)=\arcsin \mu x / 2,0 \leq x \leq 1$. As we see the function $n_{1 / 2}^{l}(s), s \in \mathbb{R}^{1}$, is bounded and real. The lemma is proved.

We see from (4.4) and (4.5) that the functions $n_{1 / 2}^{l}(s)$ and $\lambda_{1 / 2}^{l}$ are continued up to bounded, analytical functions $N^{l}(z)$ and $\Lambda^{l}(z)$ correspondingly, defined in the strip $\tilde{I}=\{z \in$ $\left.\mathbb{C}^{1}:-1 / 2 \leq \Im z \leq 1 / 2\right\}$. Let us define the functions $\widehat{N}^{l}(z)=\bar{N}^{l}(z-i / 2)$ which we shall consider in the strip $I=\{z \in \mathbb{C}: 0 \leq \Im z \leq 1\}$. The operator $\tilde{\kappa}_{1 / 2}^{l}$ coincides with the operator $\widetilde{\kappa}_{1 / 2}^{\widehat{N}^{l}}$ from the family $\left\{\widetilde{\kappa}_{\alpha}^{N^{l}}\right\}$ generated by the function $\widehat{N}^{l}$ (see (3.11)). Any other operator of this family acts as multiplication on the function:

$$
\widehat{n}_{\alpha}^{l}(s)=\left.\widehat{N}^{l}(z)\right|_{z=s+i \alpha}
$$

Denote by $\kappa_{\alpha}^{l}$ the operators

$$
\kappa_{\alpha}^{l}=\omega^{-1} \widetilde{\kappa}_{\alpha}^{N^{l}} \omega
$$

acting in $L_{2}\left(\mathbb{R}_{+}^{1}, r^{2} d r\right)$.

Note that

$$
\left(\kappa_{\alpha}^{l}\right)^{*}=\kappa_{i-\alpha}^{l} .
$$

It is convenient to represent the operator $M_{l}$ in form of three sequential maps

$$
\begin{aligned}
M_{l}: u_{0} \in \mathfrak{h}\left(u_{0}\right) & \longrightarrow \Pi^{1 / 2} u_{0}=u_{1 / 2} \longrightarrow \kappa_{1 / 2}^{l} u_{1 / 2}=v_{1 / 2} \\
& \longrightarrow \Pi^{1 / 2} v_{1 / 2}=v_{1} \in \mathfrak{h}(v)
\end{aligned}
$$

where $v=v_{0}, v_{1 / 2}, v_{1}$ are elements of the chain $\mathfrak{h}(v)$ generated by the function $\Gamma_{v}=\widehat{N}^{l} \Gamma_{u} \in \mathcal{G}$. Note that the chain (4.9) can be rewritten in the following way:

$$
u_{0} \in \mathfrak{h}\left(u_{0}\right) \stackrel{\Omega}{\rightarrow} \Gamma_{u_{0}} \longrightarrow \Gamma_{v}=\widehat{N}^{l} \Gamma_{u_{0}} \stackrel{\Omega^{-1}}{\rightarrow} \mathfrak{h}(v) \longrightarrow v_{1} \in \mathfrak{h}(v)
$$

From (4.1) and self-adjointness of $\kappa_{1 / 2}^{l}$ it follows that the operator $M_{l}$ with the domain $D\left(M_{l}\right)=V$ is symmetric. For any $\alpha \in[0,1]$, a representation of $M_{l}$ similar to (4.1) is valid:

$$
M_{l}=\Pi^{1-\alpha} \kappa_{\alpha}^{l} \Pi^{\alpha}
$$

as well as decomposition like (4.9).

Let us now describe the domain $D\left(M_{l}^{*}\right) \supseteq V$ of the operator $M_{l}^{*}$ conjugated to $M_{l}$. Let $g \in D\left(M_{l}^{*}\right)$ be a function from $D\left(M_{l}^{*}\right)$ and $h=M_{l}^{*} g \in L_{2}\left(\mathbb{R}_{+}^{1}, r^{2} d r\right)$. Then for every $u \in V=D\left(M_{l}\right)$, we can write

$$
\left(M_{l} u, g\right)=\left(\kappa_{1}^{l} \Pi u, g\right)=\left(\Pi u,\left(\kappa_{1}^{l}\right)^{*} g\right)=\left(u, \Pi \kappa_{0}^{l} g\right)=(u, h) .
$$


Here we use the representation (4.11) for $\alpha=1$ and the equality (4.8). Denote $f(r)=h(r)-$ $\left(\Pi \kappa_{0}^{l} g\right)(r)$ and apply the following evident assertion.

Lemma 4.2. Let a measurable function $f(r)$ satisfies condition

$$
\int_{0}^{\infty} f(r) u(r) r^{2} d r=0
$$

for any $u \in V$. Then $f=0$.

From this and (4.12), it follows that

$$
\Pi \kappa_{0}^{l} g=h
$$

Hence

$$
w_{0} \equiv \kappa_{0}^{l} g \in V,
$$

and $h=w_{1} \in \mathfrak{h}\left(w_{0}\right)$ is the final element of the chain $\mathfrak{h}\left(w_{0}\right)$. Thus the domain $D\left(M_{l}^{*}\right)$ of the operator $M_{l}^{*}$ is

$$
D\left(M_{l}^{*}\right)=\left\{g \in L_{2}\left(\mathbb{R}_{+}^{1}, r^{2} d r\right): \kappa_{0}^{l} g \in V\right\}
$$

In the case when the operator $\kappa_{0}^{l}$ has the inverse one, $\left(\kappa_{0}^{l}\right)^{-1}$, which is equivalent to the condition:

$$
\widehat{n}_{0}^{l}(s) \neq 0, \quad \text { for any } s \in \mathbb{R}^{1}
$$

the following equality is true:

$$
D\left(M_{l}^{*}\right)=\left(\kappa_{0}^{l}\right)^{-1} V
$$

Let $\widetilde{M}_{l}^{*}=\omega M_{l}^{*} \omega^{-1}$ be an operator in $L_{2}\left(\mathbb{R}^{1}\right)$ with domain $D\left(\widetilde{M}_{l}^{*}\right)=\omega D\left(M_{l}^{*}\right)$. Then for $\widetilde{g} \in$ $D\left(\widetilde{M}_{l}^{*}\right)$, the following representation holds true:

$$
\tilde{g}(s)=\left(\widehat{n}_{0}^{l}(s)\right)^{-1} \tilde{w}_{0}(s)=\left.\left(\widehat{N}^{l}(z)\right)^{-1} \Gamma_{w_{0}}(z)\right|_{z=s},
$$

if condition (4.17) is fulfilled. Here $\widetilde{w}_{0}(s)=\left(\omega w_{0}\right)(s)$ where $w_{0}$ is defined in (4.15).

Remarks. (1) Note that the function $\widehat{N}^{l}(z)$ is invariant with respect to reflection of the complex plane around the point $z=i / 2$ :

$$
z \longrightarrow z^{*}=-z+i
$$


Under this reflection, the strip $I$ is mapped onto itself; hence, for every zero $\bar{z} \in I(\bar{z} \neq i / 2)$ of the function $\widehat{N}^{l}$, there exists another zero, $\bar{z}^{*} \in I$, of $\widehat{N}^{l}$ with the same multiplicity. The multiplicity of $\bar{z}=i / 2=\bar{z}^{*}$ is even;

(2) Since $\widehat{N}^{l}(z) \rightarrow 2 \pi^{2} \sqrt{1-\mu^{2} / 4}>0$ as $z \rightarrow \infty$ inside $I$, the function $\widehat{N}^{l}(z)$ has finite number of zeros inside $I$.

We can now formulate the main criterion of self-adjointness of the operator $M_{l}$.

Theorem 4.3. The operator $M_{l}$ is self-adjoint if and only if the function $\widehat{N}^{l}(z)$ has no zeros in the closed strip $I$.

Proof. (1) Assume $\widehat{N}^{l}(z) \neq 0$ in the strip $I$. Then $\left(\widehat{N}^{l}\right)^{-1}(z)$ is bounded and continuous on $I$ and analytical inside $I$. Let $\tilde{g} \in \tilde{D}\left(\widetilde{M}_{l}^{*}\right)$. Since $\widehat{n}^{l}(s) \neq 0$ for $s \in \mathbb{R}^{1}$, the representation (4.19) holds true. Since

$$
\left(\widehat{N}^{l}(z)\right)^{-1} \Gamma_{w_{0}}(z)=\Gamma_{v} \in \mathcal{G}, \quad v \in V,
$$

the element $g=\omega^{-1} \tilde{g} \in D\left(M_{l}^{*}\right)$ coincides with $v \in V$, that is, $D\left(M_{l}^{*}\right)=V=D\left(M_{l}\right)$; it means the self-adjointness of $M_{l}$;

(2) assume now the function $N^{l}(z)$ has zeros $\bar{z}_{1}, \ldots, \bar{z}_{k} \in I$. Consider first the case when all zeros are lying inside $I$ and their multiplicities are equal to $p_{1}, \ldots, p_{k}$, respectively. Again, let $\widetilde{g} \in \widetilde{D}\left(\widetilde{M}_{l}^{*}\right)$. Since $\widehat{n}^{l}(s) \neq 0$, the representation (4.19) holds true. The function $\left(\widehat{N}^{l}(z)\right)^{-1} \Gamma_{w_{0}}(z)$ is meromorphic in $I$ with poles $\bar{z}_{1}, \ldots, \bar{z}_{k}$ having the order $p_{1}, \ldots, p_{k}$ respectively. For this function the usual canonical representation [10] is true:

$$
\left(\widehat{N}^{l}(z)\right)^{-1} \Gamma_{w_{0}}(z)=L^{w_{0}}(z)+\sum_{n=1}^{k} \sum_{m=1}^{p_{n}} \frac{b_{m}^{(n)}\left(w_{0}\right)}{\left(z-z_{n}\right)^{m}}
$$

where $L^{w_{0}}(z)$ is bounded, continuous function on $I$, and analytical inside $I$, and the coefficients $b_{m}^{(n)}=b_{m}^{(n)}\left(w_{0}\right)$ depend on $w_{0}$.

Lemma 4.4. The function $L^{w_{0}}(z)$ in (4.22) belongs to the space $\mathcal{G}$.

The proof of this lemma is given in The appendix.

From (4.19) and (4.22), for $g=\omega^{-1} \widetilde{g} \in D\left(M_{l}^{*}\right)$, we have

$$
g(r)=v(r)+\sum_{m, n} b_{m}^{(n)}\left(\omega^{-1}\left(\left(\frac{1}{\cdot-z_{n}}\right)^{m}\right)\right)(r)
$$

where the function $v \in V$ is defined from relation

$$
\begin{gathered}
L^{w_{0}}(z)=\Gamma_{v}(z) \in \mathcal{G}, \\
d_{m, n}(r):=\omega^{-1}\left(\left(\frac{1}{\left(\cdot-z_{n}\right)}\right)^{m}\right)(r)=A_{m}^{(n)} r^{-3 / 2-t_{n}+i s_{n}}(\ln r)^{m-1} \chi(r),
\end{gathered}
$$


where $A_{m}^{(n)}$ is an absolute constant, $z_{n}=s_{n}+i t_{n}, 0<t_{n}<1$ and

$$
X(r)= \begin{cases}1, & r>1 \\ 0, & r \leq 1\end{cases}
$$

Since linearly independent functions $d_{m, n} \in D\left(M_{l}^{*}\right)$ do not belong to $V$, due to (4.23), they form the basis in the defect subspace $\mathfrak{V}$ of the operator $M_{l}$ (see [7]). Since the dimension of the subspace $\mathfrak{V}$ is equal to $\sum_{1}^{k} p_{n}$ and the operator $M_{l}$ is real, its deficiency indexes $n_{ \pm}$are equal and have the form:

$$
n_{+}=n_{-}=\frac{1}{2} \sum_{1}^{k} p_{n}
$$

(It follows from Remarks that the sum $\sum_{1}^{k} p_{n}$ is even). Consider now the case when one of the zeros of $N^{l}(z)$, say, $\bar{z}_{0}=s_{0} \in \mathbb{R}^{1}$, lies on the boundary of $I$ and has multiplicity $p$ (in addition, there is a zero $\left.\bar{z}_{0}^{*}=s_{0}+i\right)$. In this case, in a neighborhood of $\bar{z}_{0}$, the function $\widehat{N}^{l}(z)$ has the form:

$$
\widehat{N}^{l}(z)=\left(z-\bar{z}_{0}\right)^{p} Q(z)
$$

where $Q(z)$ is analytic in this neighborhood. Consider the function,

$$
G(z)=\frac{1}{\left(-i\left(z-\bar{z}_{0}\right)\right)^{1 / 3}} \frac{1}{(z+2 i)^{2}}
$$

whereby $(-i w)^{1 / 3}$ for $\Im w>0$, we mean the branch of a many-valued function $(-i w)^{1 / 3}$ that takes positive values on the positive part of the imaginary axis. Evidently, the function $G(z)$ is analytic in the strip $I$ and satisfies condition (3.8). However, this function is discontinuous at $\bar{z}_{0}$ and does not belong to $\mathcal{G}$. In addition, the function $\widehat{N}_{(\mathrm{z})}^{l} G(z)$ now belongs to $\mathcal{G}$ as follows from (4.27) and (4.28). Thus

$$
\tilde{g}(s)=\left.G(z)\right|_{z=s} \bar{\in} \tilde{V}=\omega V,
$$

but

$$
\widehat{n}^{l}(s) \tilde{g}(s)=\left.\widehat{N}^{l}(z) G(z)\right|_{z=s} \in \tilde{V}
$$

Consequently, $g=\omega \tilde{g} \bar{\in}$ but $\kappa_{0}^{l} g \in V$, that is, $g \in D\left(M_{l}^{*}\right)$. Thus $D\left(M_{l}^{*}\right) \neq V$, and the operator $M_{l}$ has nonzero deficiency indexes. Theorem 4.3 is proved.

\section{The Operators $M_{l}$ in the Cases $l=0$ and $l=1$}

Here, we apply Theorem 4.3 to the cases $l=0$ and $l=1$. 
Theorem 5.1. (1) For $l=0$, the operator $M_{l=0}$ is self-adjoint for any $m>0$;

(2) the operator $M_{l=1}$ is self-adjoint for $m>m_{0}$ and has nonzero deficiency indexes for $m \leq m_{0}$. In addition, for $m<m_{0}$ these indexes are equal to $(1,1)$. The constant $m_{0}$ is a unique zero of $(5.4)$.

Proof. We need the following properties of the functions $\widehat{\Lambda}^{l=0}(z)$ and $\widehat{\Lambda}^{l=1}(z), z \in I$.

Lemma 5.2. (1) For any $l=0,1,2, \ldots$ the function $\widehat{\Lambda}^{l}(z)$ is invariant with respect to reflection (4.20);

(2) The point $z=i / 2 \in I$ is a nondegenerate critical point for both functions $\widehat{\Lambda}^{l=0}$ and $\widehat{\Lambda}^{l=1}$;

(3) These functions take real values on the line:

$$
\widehat{\xi}_{1 / 2}=\left\{z=s+\frac{i}{2}, s \in \mathbb{R}^{1}\right\}
$$

and on the segment:

$$
\widehat{\tau}=\{z=i t, 0 \leq t \leq 1\} .
$$

Outside the set $B=\widehat{\xi}_{1 / 2} \cup \widehat{\tau}$, both functions take nonreal values;

(4) the real values of $\widehat{\Lambda}^{l}, l=0,1$, are between 0 and $\widehat{\Lambda}^{l}(0)=\widehat{\Lambda}^{l}(i)$. Every value of $\left.\widehat{\Lambda}^{l}\right|_{B}$ except $\widehat{\Lambda}^{l}(i / 2)$-is taken exactly at two points;

(5) the extreme values of $\widehat{\Lambda}^{l}, l=0,1, \widehat{\Lambda}^{l}(0)=\widehat{\Lambda}^{l}(i)$ are given by

$$
\begin{aligned}
& \widehat{\Lambda}^{l=0}(0)=8 \sqrt{2} \pi^{2} \mu^{-1} \sin \left(\frac{1}{2} \arcsin \frac{\mu}{2}\right)>0, \\
& \widehat{\Lambda}^{l=1}(0)=-\frac{32}{3} \sqrt{2} \pi^{2} \mu^{-2} \sin ^{3}\left(\frac{1}{2} \arcsin \frac{\mu}{2}\right) \equiv-q(\mu)<0,
\end{aligned}
$$

(6) the function $q(\mu)$ increases monotonically on the interval $0<\mu<2$.

The proof of this lemma is given in The appendix.

Corollary 5.3. (1) The zeros of $\widehat{N}^{l}(z), l=0,1$ can only lie in the set $B$;

(2) $\left.\widehat{N}^{l=0}(z)\right|_{B}>0$ for any value of $\mu$, and therefore the operator $M_{l=0}$ is self-adjoint for all $m \in(0,2)$;

(3) The function $\left.\widehat{N}^{l=1}(z)\right|_{B}$ is positive if $2 \pi^{2} \sqrt{1-\mu^{2} / 4}>q(\mu)$ and vanishes at some point $z \in B$ (and also at $z^{*} \in B$ ) if $2 \pi^{2} \sqrt{1-\mu^{2} / 4} \leq q(\mu)$.

In Figure 1 , the curves corresponding to the functions $2 \pi^{2} \sqrt{1-\mu^{2} / 4}$ and $q(\mu)$ are depicted. We see that they intersect at a unique point with abscissa $\mu_{0} \in(0,2)$ which satisfies the following equation:

$$
2 \pi^{2} \sqrt{1-\frac{\mu_{0}^{2}}{4}}=q\left(\mu_{0}\right)
$$

Thus, for $m>m_{0}=2 / \mu_{0}-1$ the operator $M_{l=1}$ is self-adjoint, and for $m<m_{0}$ it has deficiency indexes $(1,1)$. For $m=m_{0}$, the operator $M_{l=1}$ is not self-adjoint as well. Theorem 5.1 is proved. 


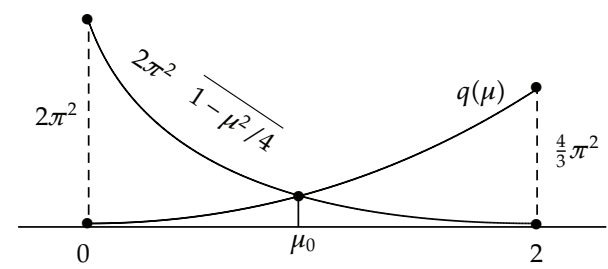

Figure 1

\section{Appendix}

Proof of Lemma 4.4. The function $\left(\widehat{N}^{l}(z)\right)^{-1}, z \in I$ admits the canonical representation (see [10])

$$
\left(\widehat{N}^{l}(z)\right)^{-1}=Q_{l}(z)+\sum_{n=1}^{k} \sum_{m=1}^{p_{n}} \frac{a_{m}^{(n)}}{\left(z-\bar{z}_{n}\right)^{m}}
$$

where $\bar{z}_{1}, \ldots, \bar{z}_{k} \in I$ are zeros of $\widehat{N}^{l}(z)$ (with multiplicities $p_{1}, \ldots, p_{k}$ ), $a_{m}^{(n)}$ are constants, $a_{p_{n}}^{(n)} \neq 0$, and $Q_{l}(z)$ is a bounded, continuous analytic function in $I$. From this, it follows that for any $v \in V, Q_{l}(z) \Gamma_{v}(z) \in \mathcal{G}$. Consider some term of the sum (A.1) and write

$$
\frac{a_{m}^{(n)}}{\left(z-\bar{z}_{n}\right)^{m}} \Gamma_{v}(z)=\left(P_{m, v}^{(n)}(z)+\sum_{d=1}^{m} \frac{c_{m-d}^{(n)}}{\left(z-\bar{z}_{n}\right)^{d}}\right) a_{m}^{(n)},
$$

where

$$
\begin{gathered}
P_{m, v}^{(n)}(z)=\frac{1}{\left(z-\bar{z}_{n}\right)^{m}}\left(\Gamma_{v}(z)-\sum_{d=1}^{m} c_{m-d}^{(n)}\left(z-\bar{z}_{n}\right)^{m-d}\right), \\
c_{t}^{(n)}=c_{t}^{(n)}(v)=\frac{1}{t !} \Gamma_{v}^{(t)}\left(\bar{z}_{n}\right), \quad t=0,1, \ldots
\end{gathered}
$$

It is clear that $P_{m, v}^{(n)}(z)$ is bounded, continuous analytic function in $I$. We are going to show that this function belongs to $\mathcal{G}$. Let $O \in I$ be a small neighborhood of $\bar{z}_{n}$ and $\chi_{O}(z)$ the characteristic function of $O$. Obviously, the bounded function $\chi_{O} P_{m, v}^{(n)}$ satisfies condition (3.8). Every term of the sum

$$
\left(1-\chi_{O}\right) P_{m, v}^{(n)}(z)=\frac{\Gamma_{v}(z)}{\left(z-\bar{z}_{n}\right)^{m}}\left(1-\chi_{O}\right)-\sum_{d=1}^{m} \frac{c_{m-d}^{(n)}(v)}{\left(z-\bar{z}_{n}\right)^{d}}\left(1-\chi_{O}\right)
$$

satisfies this condition as well. 
Thus for fixed $\bar{z}_{n}$ and $v \in V$,

$$
\sum_{m=1}^{p_{n}} \frac{a_{m}^{(n)} \Gamma_{v}(z)}{\left(z-\bar{z}_{n}\right)^{m}}=K_{v}^{(n)}(z)+\sum_{d=1}^{p_{n}} \frac{b_{d}^{(n)}(v)}{\left(z-\bar{z}_{n}\right)^{d}},
$$

where

$$
\begin{gathered}
K_{v}^{(n)}(z)=\sum_{m=1}^{p_{n}} a_{m}^{(n)} P_{m, v}^{(n)}(z), \\
b_{d}^{(n)}(v)=\sum_{m=1}^{p_{n}} a_{m}^{(n)} c_{m-d}^{(n)}(v), \quad d=1, \ldots, p_{n} .
\end{gathered}
$$

Thus, we get the representation (4.22) where

$$
L^{\left(w_{0}\right)}(z)=Q_{l}(z) \Gamma_{w_{0}}(z)+\sum_{n=1}^{k} K_{w_{0}}^{(n)}(z) \in \mathcal{G},
$$

and the coefficients $b_{m}^{(n)}\left(w_{0}\right)$ are given by formula (A.7). Lemma 4.4 is proved.

Proof of Lemma 5.2. (1) It is more convenient to consider the functions $N^{l}(z)$ and $\Lambda^{l}(z)$ in the strip $\widetilde{I}=\{z:|\Im z|<1 / 2\}$ instead of the functions $\widehat{N}^{l}(z)$ and $\widehat{\Lambda}^{l}(z)$ in the strip $I$. Similarly, instead of the reflection $z \rightarrow z^{*}$ we consider the reflection $z \rightarrow-z$ around the point $z_{0}=0$. It is clear that the functions $\Lambda^{l}(z), l=0,1,2, \ldots$ are invariant with respect to the change $z \rightarrow-z$, and it means the invariance of $\hat{\Lambda}^{l}$ with respect to reflection (4.20);

(2) it follows from (4.5) that $z=0$ is a nondegenerated critical point of $\Lambda^{l=0}$ and $\Lambda^{l=1}$, if we note that $0<v(x) \leq \pi / 2$. Correspondingly, $z=i / 2$ is a nondegenerated critical point for $\widehat{\Lambda}^{l}(z), l=0,1$. The real axis $\xi_{0}=\left\{z=s ; s \in \mathbb{R}^{1}\right\}$ coincides with the saddle-point line at $z=0$ (see [10]) for $\Lambda^{l=0}$ and $-\Lambda^{l=1}$. More precisely, these functions take real values on $\xi_{0}$ and decrease monotonically to zero as $|s|$ increases from zero to infinity. On the contrary, $\Lambda^{l=0}$ and $-\Lambda^{l=1}$ increase monotonically along imaginary axis as $|t|$ increases from zero to $1 / 2$. The monotonicity of $\Lambda^{l=0}$ along real axis follows from (4.5), equality $P_{0}(x) \equiv 1$, and inequality

$$
\left(\frac{\operatorname{ch}(v(x) s)}{\operatorname{ch}((\pi / 2) s)}\right)_{s}^{\prime}<-\frac{\pi}{2} \frac{\operatorname{sh}(\pi / 2-v(x)) s}{(\operatorname{ch}((\pi / 2) s))^{2}}<0
$$

for $s>0$ and a similar inequality for $s<0$. The proof of monotonicity of $\Lambda^{l=1}$ along real axis, and also monotonicity of both functions along imaginary axis is analogous if we note that $P_{l=1}(x) \equiv x$ on $(0,1)$. Thus the functions $\Lambda^{l}, l=0,1$, take all values between 0 and $\Lambda^{l}(i / 2)=$ $\Lambda^{l}(-i / 2)$ and every value except $\Lambda^{l}(0)$ which is taken exactly twice;

(3) we will show now that the values of functions $\Lambda^{l}(z), l=0,1$, on the set $\tilde{I} \backslash B$ are nonreal. Let us represent this set as a union of four sets, $\widetilde{I}_{i}, i=1,2,3,4$ as shown in Figure 2.

We consider the case $l=0$; the case $l=1$ is similar. Figure 3 shows the disposition of lines of levels for function $K_{0}(z)=\Re \Lambda^{l=0}(z)$ which pass through the points $i$ and $-i$ between lines $\beta$ and $\beta^{*}, \beta=\left\{z: K_{0}(z)=0, \Im z>0\right\}, \beta^{*}=\left\{z: K_{0}(z)=0, \Im z<0\right\}$. 


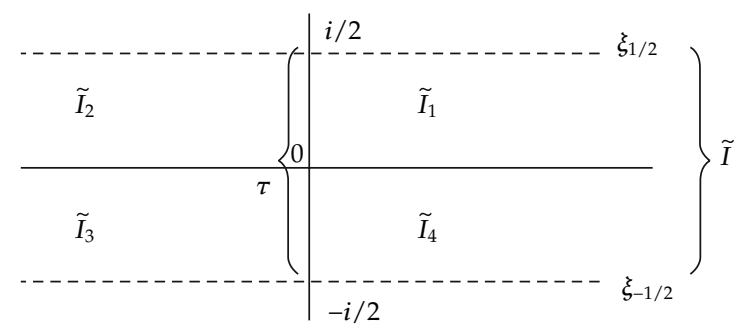

Figure 2

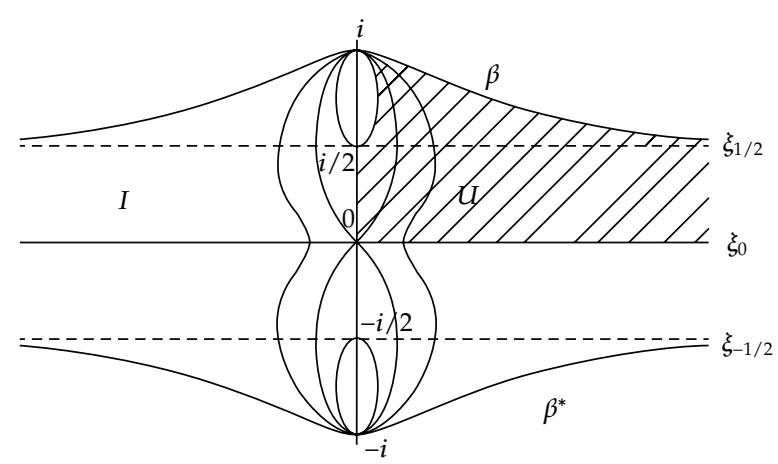

Figure 3

All these lines have common tangents at points $i$ and $-i$, and the line $\beta$ (resp. $\beta^{*}$ ) lies above (resp., below) the strip $\widetilde{I}$. The picture represented in Figure 3 is obtained by detailed study of the explicit formula for $\Lambda^{l=0}$ :

$$
\Lambda^{l=0}(z)=\frac{4 \pi^{2}}{\mu} \frac{\operatorname{sh}(z \arcsin (\mu / 2))}{z \operatorname{ch}(z \cdot \pi / 2)}
$$

together with the proof that the lines $\beta$ and $\beta^{*}$ do not intersect the strip $\widetilde{I}$. This proof is given below.

From Figure 3, we see that the set $I_{1}$ lies inside the shaded domain $U$ that is bounded by the real semiaxis $\xi_{0}^{+}=\{z: z=s, s>0\}$, the segment $(0, i / 2)$ on the imaginary axis and the part of line $\beta$ which lies in the right half-plane. From (A.10), it is easy to see that the function $w=\Lambda^{l=0}(z)$ maps the boundary $\partial U$ of the domain $U$ into the boundary of the right lower quadrant $M=\{w: \Re w>0, \Im w<0\}$ of the plain $w$. Hence, the domain $U$ is mapped inside this quadrant, that is, all values of the function $\Lambda^{l=0}$ in $U$ are nonreal. It means the absence of real values of $\Lambda^{l=0}$ in $\widetilde{I}_{1}$. For the domains $\widetilde{I}_{2}, \widetilde{I}_{3}$, and $\widetilde{I}_{4}$, the proof is similar. Let us now prove that $\beta$ and $\beta^{*}$ do not intersect the line $\xi_{1 / 2}$. It is sufficient to prove that $\Re \Lambda^{l=0}>0$ on the line $\xi_{1 / 2}=\left\{z: z=s+i / 2, s \in \mathbb{R}^{1}\right\}$ or, which is the same, that

$$
\left.\mathfrak{R} \frac{\operatorname{ch}(z v(x))}{\operatorname{ch}(z \pi / 2)}\right|_{z=s+i / 2}>0,
$$


for any $s \in \mathbb{R}^{1}$ and $x \in(0,1)$. Write

$$
\frac{\operatorname{ch}[(s+i / 2) v(x)]}{\operatorname{ch}[(s+i / 2) \pi / 2]}=\frac{\operatorname{ch}(s v(x)) \cos (v(x) / 2)+i \operatorname{sh}(s v(x)) \sin (v(x) / 2)}{\operatorname{ch}(s \pi / 2) \cos (\pi / 4)+i \operatorname{sh}(s \pi / 2) \sin (\pi / 4)}=D(s, x) .
$$

Let $s>0$. Then the values of numerator and denominator of $D(s, x)$ lie in the right upper quadrant of a complex plain, and hence $-\pi / 2<\arg D(s, x)<\pi / 2$, that is, $\mathfrak{R} D(s, x)>$ 0 . Similarly (A.11) can be proved in the case $s<0$ and for $\left.\Lambda^{l=0}\right|_{z=s-i / 2}$;

(4) let us find the values $\Lambda^{l}(i / 2), l=0,1$ :

(I) the case $l=0$ :

$$
\Lambda^{l=0}(i / 2)=2 \pi^{2} \int_{0}^{1} \frac{\cos (v(x) / 2)}{\cos v(x) \cos (\pi / 4)} d x
$$

After the change $v(x)=\xi$, the integral (A.13) becomes

$$
\frac{4 \sqrt{2} \pi^{2}}{\mu} \int_{0}^{\arcsin \mu / 2} \cos \left(\frac{\xi}{2}\right) d \xi=\frac{8 \sqrt{2}}{\mu} \pi^{2} \sin \left(\frac{1}{2} \arcsin \frac{\mu}{2}\right) ;
$$

(II) The case $l=1$ :

$$
\Lambda^{l=1}\left(\frac{i}{2}\right)=-2 \pi^{2} \int_{0}^{1} x \frac{\sin (v(x) / 2) d x}{\cos v(x) \sin (\pi / 4)} .
$$

The same change $v(x)=\xi$ reduces to the integral

$$
-\frac{8 \sqrt{2} \pi^{2}}{\mu^{2}} \int_{0}^{\arcsin \mu / 2} \sin \xi \sin \left(\frac{\xi}{2}\right) d \xi=-\frac{32 \sqrt{2}}{3} \frac{\pi^{2}}{\mu^{2}} \sin ^{3}\left(\frac{1}{2} \arcsin \frac{\mu}{2}\right)
$$

(5) let us show that the function:

$$
q(\mu)=2 \pi^{2} \int_{0}^{1} x \frac{\sin (v(x) / 2)}{\cos v(x) \sin (\pi / 4)} d x
$$

decreases monotonically as $\mu$ changes from 0 to 2 . We have

$$
\left(\frac{\sin (v(x) / 2)}{\cos v(x)}\right)_{\mu}^{\prime} \geq 0
$$

because the numerator of (A.18) increases, while the denominator decreases with the growth of $\mu$. This implies that

$$
q^{\prime}(\mu) \geq 0,
$$

that is, $q(\mu)$ increases monotonically. Lemma 5.2 is proved. 


\section{Acknowledgment}

This work is supported by RFBR Grant 11-01-00485a.

\section{References}

[1] R. A. Minlos and M. H. Shermatov, On Pointwise Interaction of Three Particles, vol. 6, Vestnik Moskovskogo Universiteta, 1989.

[2] M. H. Shermatov, "Point interaction between two fermions and one particle of a different nature," Theoretical and Mathematical Physics, vol. 136, no. 2, pp. 257-270, 2003 (Russian).

[3] R. A. Minlos, "On point-like interaction between $n$ fermions and another particle," Moscow Mathematical Journal, vol. 11, no. 1, pp. 113-127, 2011.

[4] R. A. Minlos, "Remarks on my paper "On point-like interaction between $n$ fermions and another particle"," Moscow Mathematical Journal, vol. 11, no. 4, pp. 1-3, 2011.

[5] R. A. Minlos and L. D. Faddeev, "On the point interaction for a three-particle system in quantum mechanics," Doklady Akademii Nauk USSR, vol. 141, no. 6, pp. 1335-1338, 1961 (Russian).

[6] I. M. Gelfand, R. A. Minlos, and Z. Ya. Shapiro, Representation of Group of Rotation and Lorenz's Group, Moscow, Russia, 1958.

[7] M. A. Neimark, Linear Differential Operators, Gostechizdat, Moscow, Russia, 1954.

[8] Mathematical Encyclopedia, vol. 3, Moscow, Russia, 1982.

[9] R. Paley and N. Wiener, Fourier Transforms in Complex Domain, AMS, New York, NY, USA, 1934.

[10] B. V. Shabat, Introduction to Complex Analysis Part 1, Nauka, Moscow, Russia, 1976. 


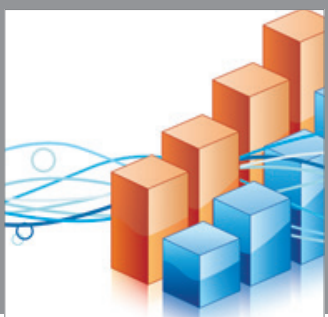

Advances in

Operations Research

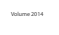

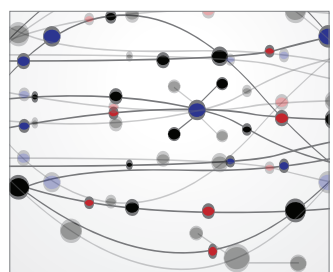

\section{The Scientific} World Journal
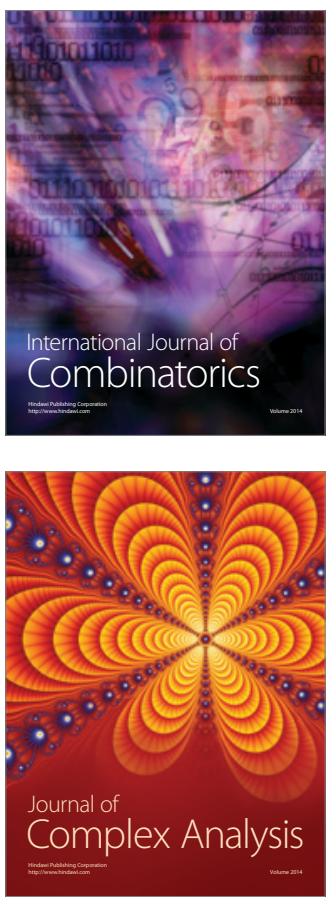

International Journal of

Mathematics and

Mathematical

Sciences
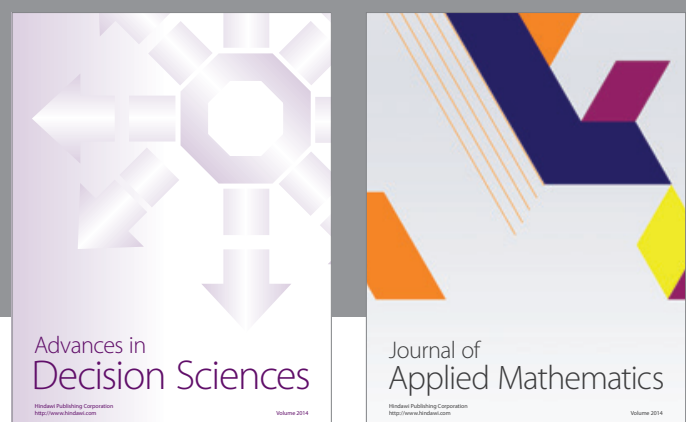

Journal of

Applied Mathematics
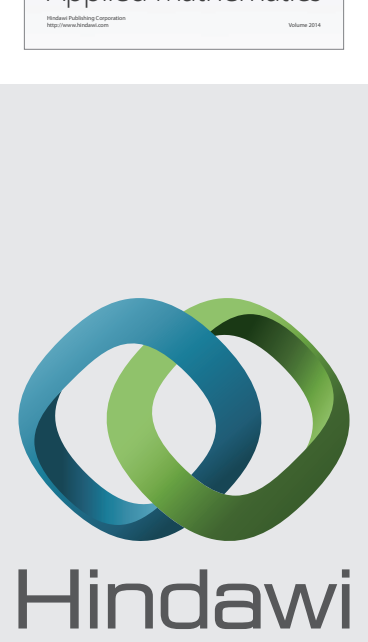

Submit your manuscripts at http://www.hindawi.com
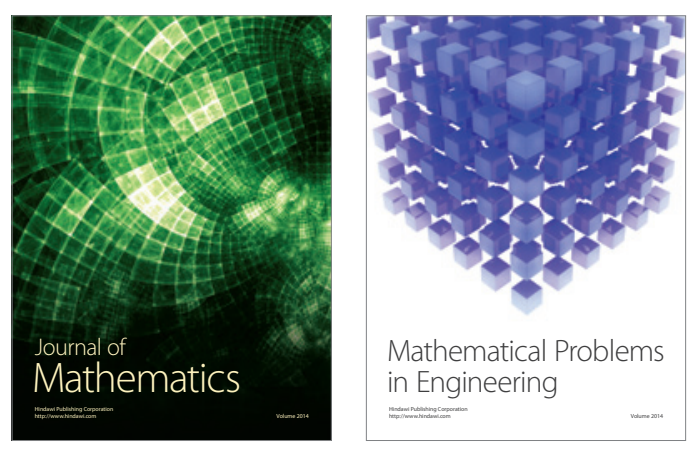

Mathematical Problems in Engineering
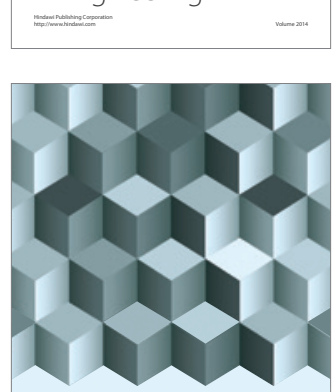

Journal of

Function Spaces
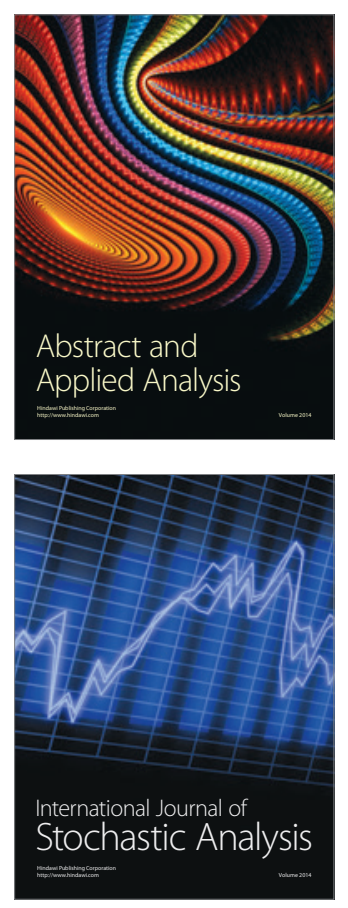

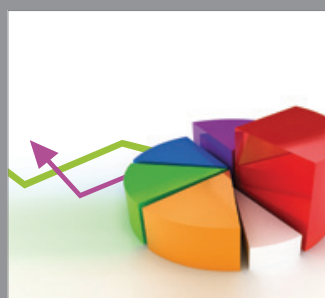

ournal of

Probability and Statistics

Promensencen
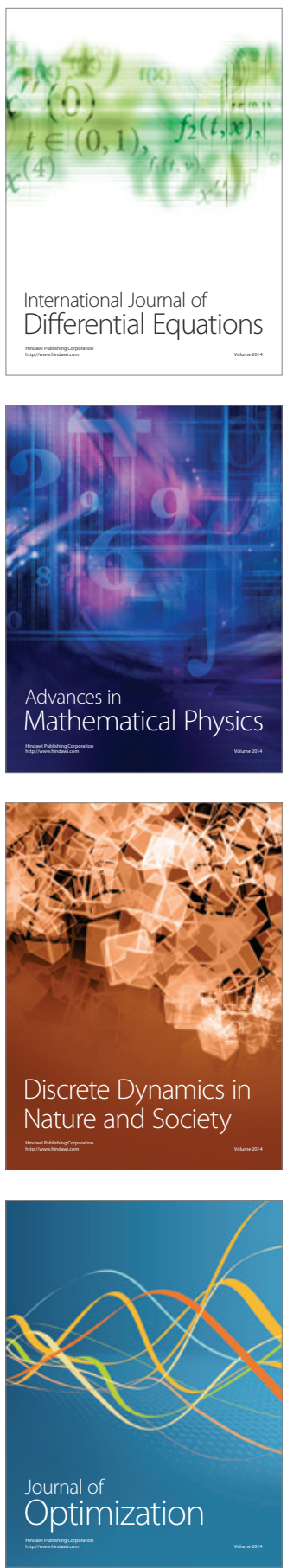